\title{
Funding Source for Product
}

National Cancer Institute

\section{Source}

National Cancer Institute. Funding Source for Product. NCI Thesaurus. Code C102431.

Source of money used to acquire the product. 